\title{
Ultra-wide coverage VLC system with alignment-free receiver
}

Crisanto Quintana, Hyunchae Chun, Rahmat Mulyawan, Grahame Faulkner, Steve Collins, Dominic C. O'Brien, Department of Engineering Science, University of Oxford, Oxford OXI 3PJ, UK. e-mail: hyunchae.chun@eng.ox.ac.uk

Enyuan Xie, Jonathan J. D. McKendry, Erdan Gu, Martin D. Dawson, Department of Physics, Institute of Photonics, University of Strathclyde, Glasgow G1 1RD, UK.

Pavlos P. Manousiadis Dimali A. Vithanage, Graham A. Turnbull, Ifor D.W. Samuel Organic Semiconductor Centre, SUPA, School of Physics and Astronomy, University of St Andrews, St Andrews KY16 9SS, UK.

\begin{abstract}
In this work we present an ultra-wide coverage visible light communication (VLC) system based on a mechanical beam steering system at the transmitter terminal and a fluorescent optical concentrator at the receiver. The transmitter and receiver full field of view (FFOV) are $100^{\circ}$ and $120^{\circ}$, respectively. The coverage area of the transmitter system at $2 \mathrm{~m}$ is $12.5 \mathrm{~m}^{2}$ and a data link of $300 \mathrm{Mbps}$ has been achieved at this range.
\end{abstract}

\section{1) Introduction}

Visible light communication (VLC) has emerged as a promising solution to overcome the saturation of $\mathrm{RF}$ spectrum and it provides $\sim \mathrm{THz}$ of unlicensed bandwidth [1]. Research conducted during the last decade has led to a wide range of white optical sources that are able to combine both illumination and very high speed switching times [2]. Multi Gigabits data links has been reported using light emitting diodes (LEDs) [2-4], often using narrow beams of light between the transmitter and receiver. However, in certain indoors applications, such as video virtual reality (VR) and augmented reality (AR), it is essential to provide high mobility (i.e. alignment-free coverage) to the user.

In this work, we present the design and implementation of a novel ultra-wide coverage VLC system that offers full field of views (FFOV) above $100^{\circ}$ at both transmitter and receiver. A reliable data link has been achieved at $300 \mathrm{Mbps}$. The rest of the paper is structured as follows. The system architecture and the detailed design of transmitter and receiver modules are described in section 2. Experimental results are then presented in section 3. Finally, conclusions drawn from this work and future work are presented in section 4 .

\section{2) System architecture}

Figure 1 shows the system architecture. At the base station terminal (transmitter) a wide angle steering system will direct the light towards the user terminal (receiver). At the receiver terminal, the light is detected with a wide angle of acceptance detector, which is based on a fluorescent optical concentrator and a high speed photodetector. Both transmitter and receiver terminals detailed block diagrams are shown in figure 2 and described in the following subsections. 


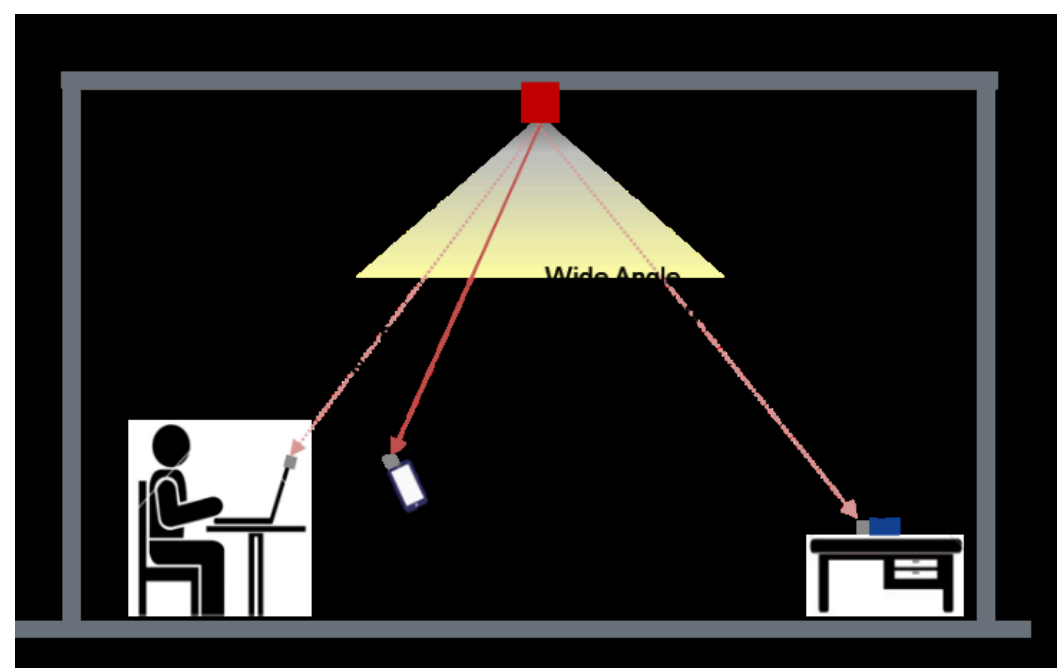

Figure 1. Schematic diagram, of indoor optical wireless link with wide angle beam-steering.

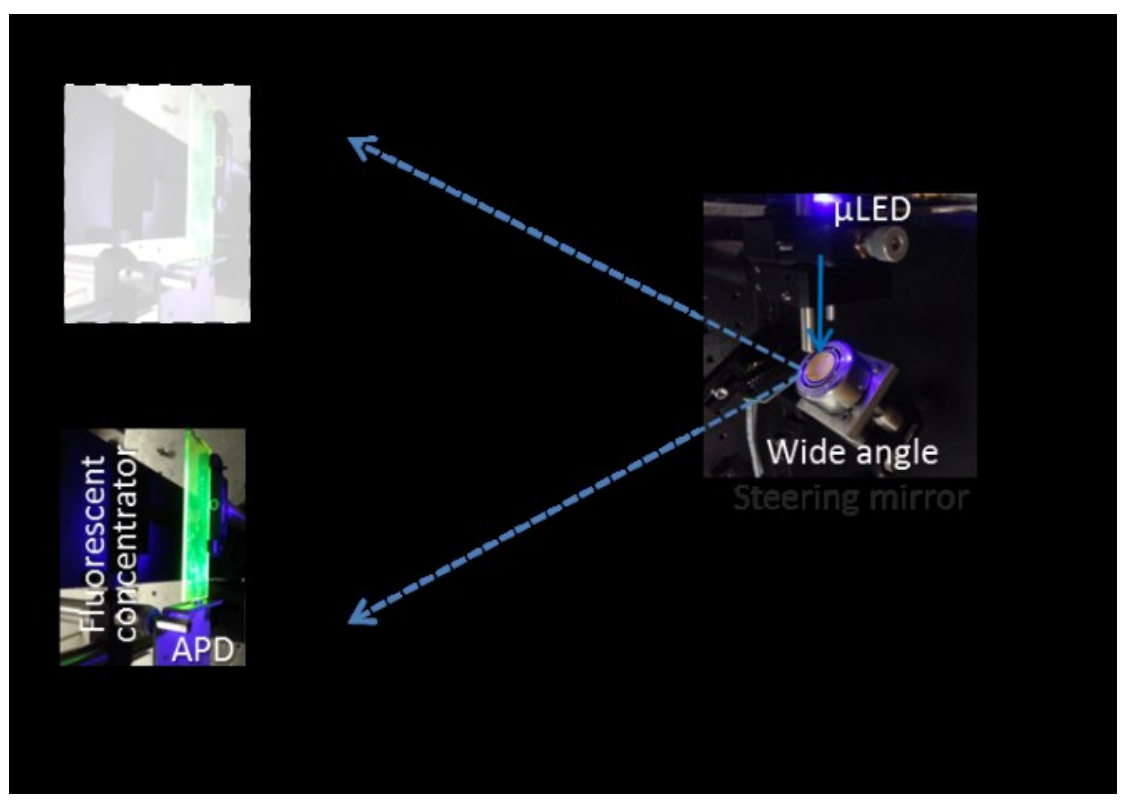

Figure 2. Schematic diagram of the experimental setup.

\section{a) Transmitter}

The transmitter is based on a blue micro-LED with a bandwidth (BW) of $80 \mathrm{MHz}$. It is modulated by an arbitrary waveform generator (Tektronix, AWG70002) with a DC bias, which generates the pseudorandom sequences used to test the data link. White light can be achieved using color converter materials or adding two or more additional LEDs to create an RGB source [5]. Micro-LEDs provide very high speed switching times $(10 \mathrm{~s} \sim 100 \mathrm{~s}$ of $\mathrm{MHz} \mathrm{BW})$ at the expense of a limited transmitted power. This power restriction makes them not suitable for diffuse links. A beam steering module is then required to provide a wide angle of coverage. In this work we used a mechanical beam steering system (dual axis steerable mirror), which provides an optical deflection of $\pm 50^{\circ}$.

\section{b) Receiver}

The receiver consists of a fluorescent optical concentrator and a high speed avalanche photodetector (Hamamtsu C12702). This topology breaks the conservation of etendue allowing a receiver with both large FOV and collection area [6-7]. The optical concentrator was fabricated with a very thin layer of 
fluorescent material (Coumarin 6) embedded between two microscope slides. Some of the blue light incident on the fluorescent optical concentrator is absorbed by the fluorophore and reemitted at a longer wavelength. The light is then guided by a total internal reflection (TIR) to an edge of the concentrator, where it is finally detected by the high speed avalanche photodetector. The collection area of the receiver is $19 \mathrm{~cm}^{2}(75 \times 25 \mathrm{~mm})$ and its FOV $60^{\circ}$. This large angle of acceptance provides a link free of alignment.

The concentrated light sensed by the avalanche photodetector is amplified using a trans-impedance amplifier (TIA) and then acquired by an oscilloscope (Agilent MSO7104B). The captured signal is transferred to a computer for post-processing. A decision feedback equalizer (DFE) with 4 backwards and 10 forward taps was used to compensate for the limited BW provided by the LED and the optical concentrator.

\section{3) Results}

A data link was tested at 200,300, 400 and $500 \mathrm{Mbps}$ at 0 and $45^{\circ}$. The link bit error rate (BER) was measured using transmitting frames of 8192 bits length $\left(8 \mathrm{x} 2^{10}\right)$. Figure 3 shows three representative eye diagrams from these experiments and the measured BER at various data rates. The results show that at $0^{\circ}$, the BER is below $1.22 \times 10^{-4}$. However, at a steering angle of $45^{\circ}$, it deteriorates as the link data rate is increased. This is because of the source's divergence and the cosine law means that the light collected by the concentrator is reduced as the incident angle increases. At 300Mbps the measured BER is $1.2 \times 10^{-3}$, which is sufficiently low that error free communications can be achieved by using a state of the art forward error correction (FEC) code [8]. These codes introduce an overhead of $7 \%$ but provide error free links for raw BER below $3.8 \times 10^{-3}$.

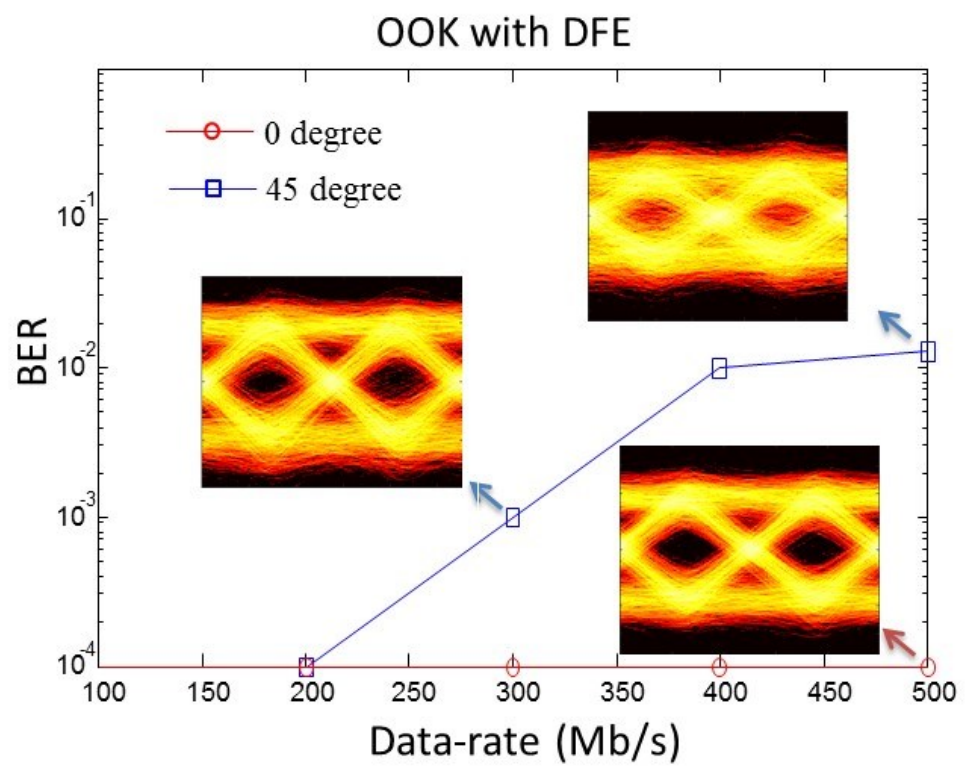

Figure 3.Representative eye diagrams and the BER (Bit error rate) results at different angles and data rates

\section{4) Conclusions}

In this work, an ultra-wide coverage indoor optical wireless communication system was described. The use of wide-angle steering mirror at the transmitter end and fluorescent optical concentrator at the receiver was demonstrated, and a data link of $300 \mathrm{Mbps}$ was achieved at a range of $2 \mathrm{~m}$ using on-offkeying. The transmitter terminal has a FFOV of $100^{\circ}$ with a maximum coverage area of $12.5 \mathrm{~m}^{2}$ at $2 \mathrm{~m}$. 
The receiver provides a large collection area of $19 \mathrm{~cm}^{2}$ and captures light falling at angles up to $\pm 60^{\circ}$. Future work includes generating white-light and increasing data capacity by optimising the transmitter and the receiver.

\section{References}

[1] Demers, H. Yanikomeroglu, and M. St-Hilaire, "A survey of opportunities for free space optics in next generation cellular networks," CNSR 2011, pp. 210-216, 2011.

[2] A. M. Khalid, G. Cossu, R. Corsini, P. Choudhury and E. Ciaramella, "1-Gb/s Transmission Over a Phosphorescent White LED by Using Rate-Adaptive Discrete Multitone Modulation," IEEE Photonics Journal, vol. 4, no. 5, pp. 1465 - 1473, Oct. 2012.

[3] H. Chun et al., "LED Based Wavelength Division Multiplexed $10 \mathrm{~Gb} / \mathrm{s}$ Visible Light Communications," in Journal of Lightwave Technology, vol. 34, no. 13, pp. 3047-3052, July1, 12016. [4] R. X. G. Ferreira et al., "High Bandwidth GaN-Based Micro-LEDs for Multi-Gb/s Visible Light Communications," in IEEE Photonics Technology Letters, vol. 28, no. 19, pp. 2023-2026, Oct.1, 1 2016.

[5] P. Manousiadis et al., "Demonstration of $2.3 \mathrm{~Gb} / \mathrm{s}$ RGB white-light VLC using polymer based colour-converters and GaN micro-LEDs," 2015 IEEE Summer Topicals Meeting Series (SUM), Nassau, 2015, pp. 222-223.

[6] Pavlos P. Manousiadis, Sujan Rajbhandari, Rahmat Mulyawan, Dimali A. Vithanage, Hyunchae Chun, Grahame Faulkner, Dominic C. O'Brien, Graham A. Turnbull, Stephen Collins, and Ifor D.W. Samuel, "Wide field-of-view fluorescent antenna for visible light communications beyond the étendue limit," Optica 3, 702-706 (2016)

[7] T. Peyronel, K. J. Quirk, S. C. Wang, and T. G. Tiecke, "Luminescent detector for free-space optical communication," Optica 3, 787-792 (2016)

[8] F. Chang, K. Onohara, and T. Mizuochi, "Forward error correction for $100 \mathrm{G}$ transport networks", IEEE Commun. Mag., vol. 48 (3), pp. S48-S55, Mar. 2010. 